\title{
A Rare Case of Secondary Cutis Verticis Gyrata on the Forehead
}

\author{
Joo Sung Jung (D), Nam Kyu Lim (1) \\ Department of Plastic and Reconstructive Surgery, Dankook University Hospital, Cheonan, Korea
}

\begin{abstract}
Cutis verticis gyrata (CVG) is an unusual morphological condition of the scalp characterized by ridges and furrows resembling the surface of the brain. Most patients have lesions on the scalp, and only a few cases with forehead lesions have been described in literature. We report an extremely rare case of secondary CVG on the forehead. A 61-year-old female patient was referred to our outpatient clinic with a large area of hypertrophic skin on the forehead. A lesion measuring $12 \times 3 \mathrm{~cm}$ extended across both eyebrows, the glabella, and the forehead. The patient reported that a pruritic erythematous lesion in that region had occurred 10 years ago after she performed acupuncture on herself on the forehead several times. She had no underlying diseases and no relevant family history. We removed the entire lesion and covered the defect with a forehead advancement flap. This solved the initial aesthetic problem. Her forehead skin became flattened, and the sagging upper eyelid skin was aesthetically corrected. Pathological findings showed nodular lesions with dense collagen fibers and microvascular proliferation, supporting the diagnosis of CVG. We achieved good results through surgical treatment for the extremely rare case of $\mathrm{CVG}$ on the forehead.
\end{abstract}

Keywords: Cutis verticis gyrata; Acupuncture; Forehead

\section{Introduction}

Cutis verticis gyrata (CVG) is a rare condition of the scalp characterized by convoluted folds and furrows. Historically, the condition was first described by Unna in 1907 and is nowadays classified by the underlying etiology as either primary or secondary [1]. Though most lesions are located in the scalp, some rare cases are also found in other parts of the body $[2,3]$. Our case involves both the forehead and glabella of the face indicating potential for facial involvement in CVG. We present a female patient with secondary CVG associated with acupuncture and discuss the diagnostic and therapeutic aspects of CVG. The patient provided written informed consent for the publication and the use of her images.

\section{Case}

A 61-year-old woman with no significant underlying condition presented as an outpatient with an irreversible wide scar on the forehead. A lesion measuring $12 \times 3 \mathrm{~cm}$ extended across both eyebrows, the glabella, and the forehead. She described a history of pruritic erythema after self-acupuncture on the forehead 10 years ago, but had no history of neuropsychiatric or ophthalmological disorders. The patient was of normal intelligence and had no family history of a similar condition. The patient reported feeling significant stress and complained that the skin change was aesthetically un-

\section{Case Report}

Received: February 8, 2020

Revised: March 6, 2020

Accepted: March 8, 2020

\section{Corresponding author:}

Nam Kyu Lim, M.D.

Department of Plastic and Reconstructive surgery, Dankook University Hospital, 201

Manghyang-ro, Dongnam-gu, Cheonan 31116, Korea

Tel: +82-41-550-6477

Fax: +82-41-554-6477

E-mail: linakyer@daum.net

This study was presented at PRS Korea 2019 on November 8-10, in Seoul, Korea.

This is an Open Access article distributed under the terms of the Creative Commons Attribution Non-Commercial License (https://creativecommons.org/licenses/by-nc/4.0/) which permits unrestricted non-commercial use, distribution, and reproduction in any medium, provided the original work is properly cited.

(c) 2020 Korean Wound Management Society 

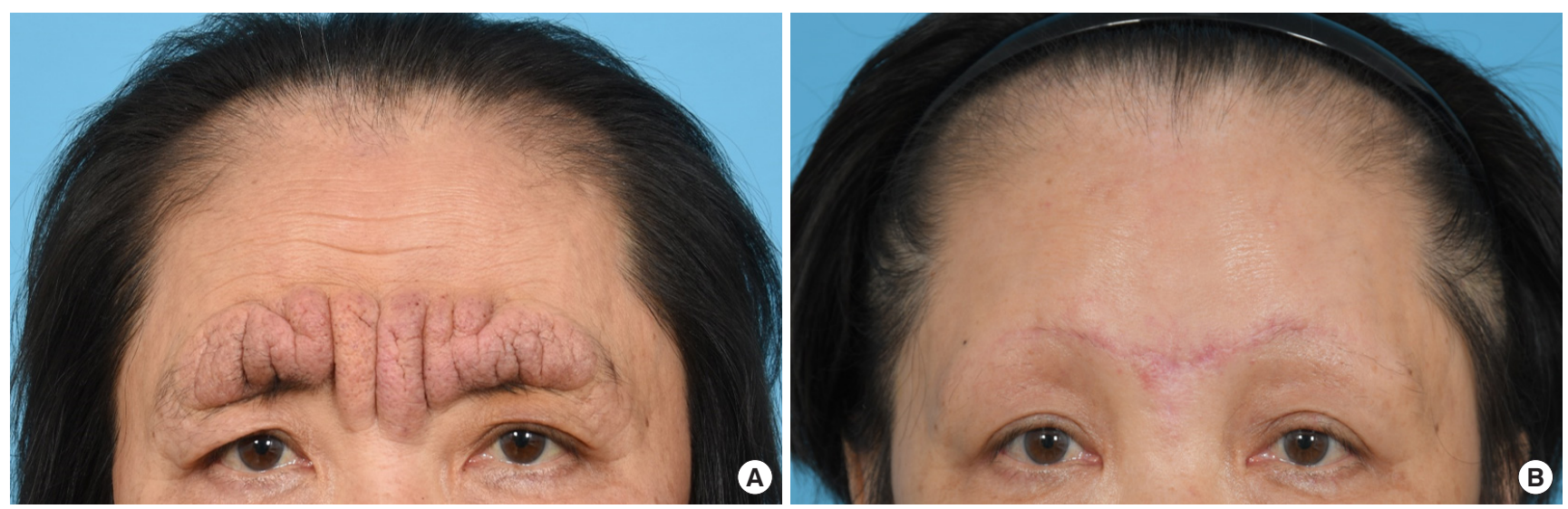

Fig. 1. The clinical images of the patient. (A) The preoperative image. (B) Image from 6 months after surgery.
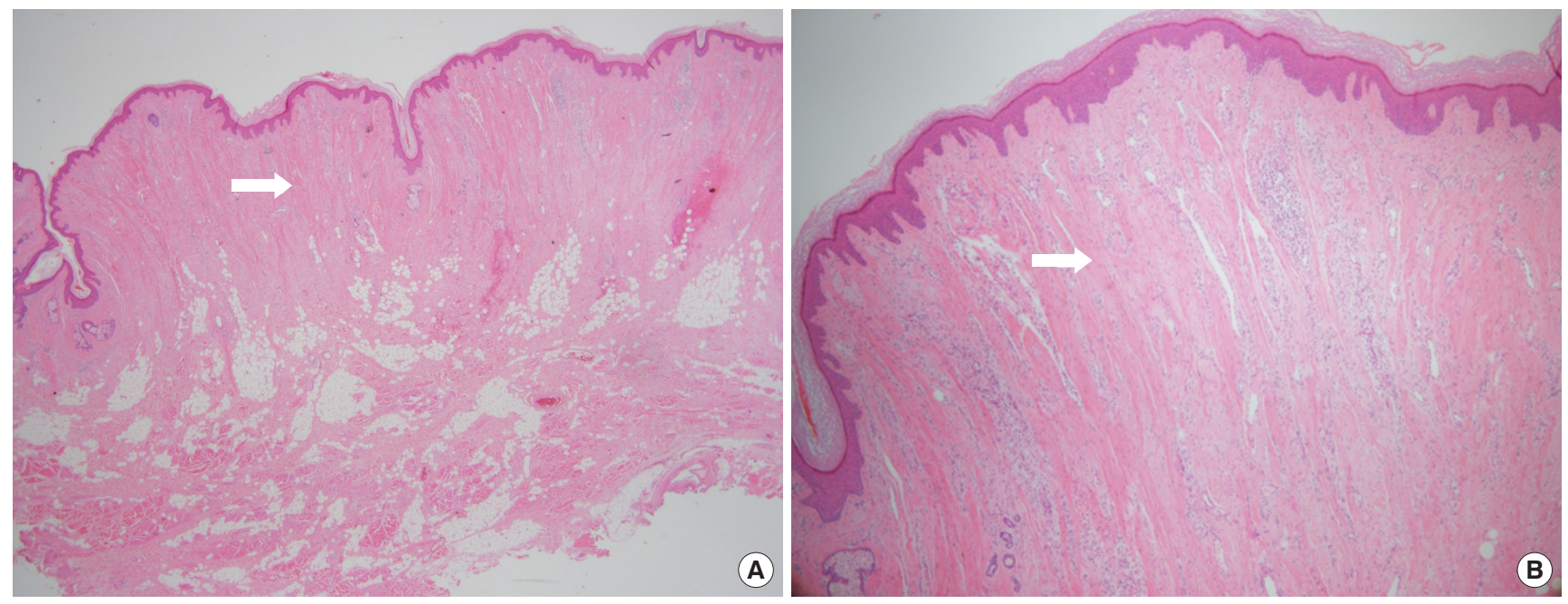

Fig. 2. The pathologic images of the patient. (A) Dense collagen fibers (arrow, H\&E, $\times 20$ ). (B) Microvascular proliferation (arrow, H\&E, $\times 40)$.

pleasing. Hypertrophy and folding of the skin were observed, suggesting a diagnosis of CVG (Fig. 1A). No signs of skin changes on the trunk, limbs, or scalp were observed. An initial 4-mm punch biopsy performed before surgery showed nodular lesions with dense collagen fibers oriented vertically to the skin surface and with microvascular proliferation (Fig. 2). Magnetic resonance imaging showed widely thickened subcutaneous tissue in the forehead and glabella (Fig. 3). However, laboratory findings-including insulin-like growth factor (IGF)-1 and IGF-binding protein-3 levels-showed no unusual elevations, and all blood test parameters were within the normal range. Based on the patient's clinical presentation and histological results, the diagnosis of secondary CVG was made. We excised the area affected by CVG and released the corrugator supercilii and procerus muscles. During the proce-

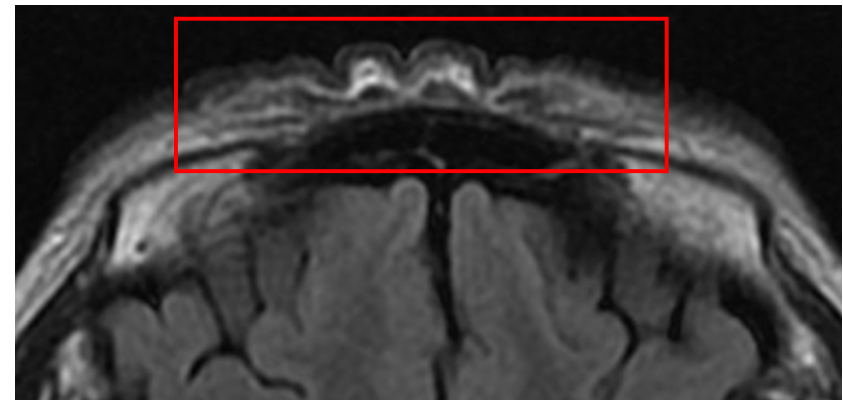

Fig. 3. The magnetic resonance imaging of cutis verticis gyrata. Note convoluted skin furrows and thickened subcutaneous tissue (red box).

dure, the supratrochlear and supraorbital neurovascular bundles were preserved. We closed the defect site with an advanced forehead flap that contained the frontalis muscle. After 
layered closure, the medial epicanthal fold was pulled upward due to tension in the nasion and nasal dorsum area. Therefore, we performed a medial epicanthoplasty to prevent this from occurring. Postoperatively, the patient received daily dressings of the suture site and intravenous antibiotics, and was discharged on postoperative day 2. A microscopic examination of the surgical specimen confirmed the previous findings (Fig. 2). At a 1-week follow-up, the patient was healing well without any complications. No additional surgery or medication was needed, and there was no recurrence throughout 6 months of follow-up (Fig. 1B). The size of the gyrata was dramatically reduced, and only mild hyperemic scars were visible. The patient was satisfied with her aesthetic outcome, and is receiving monthly laser therapy for scar management.

\section{Discussion}

The term "cutis verticis gyrata" is a descriptive diagnosis of an uncommon type of lesion that is characterized by convoluted skin folds and furrows, which are mainly localized in the scalp. The prevalence of CVG is 1 in 100,000, and CVG primarily causes aesthetic problems, rather than functional issues [1]. In rare cases, CVG occurs in locations other than the scalp, although only a few such cases have been reported in the literature $[2,4]$.

Although researchers have proposed diverse ways of classifying this unusual disease, the most useful system of classifying CVG has proven to be a distinction between primary and secondary [5]. In primary CVG, which sometimes occurs in association with neuropsychiatric conditions such as epilepsy, cognitive delays, and schizophrenia, the skin shows normal histopathological findings. Secondary CVG occurs more frequently than primary CVG, and it is associated with a broader range of diseases. For instance, local neoplastic conditions with scalp involvement, such as fibromas, congenital cerebriform intradermal nevus, and other hamartomas, have been proposed to play a causative role in the development of CVG. Furthermore, the distinctive folds that are characteristic of CVG can result from inflammatory conditions affecting the scalp, including erysipelas, impetigo, eczema, and psoriasis. The characteristic histopathological findings of secondary CVG include increased levels of ground substance, collagen, and fibroblasts, as well as enlarged epidermal appendages [1]. More generally, however, the histopathological features of secondary CVG are reflective of the underlying disease [5]. Since the diagnosis of CVG is based on a combination of clinical and

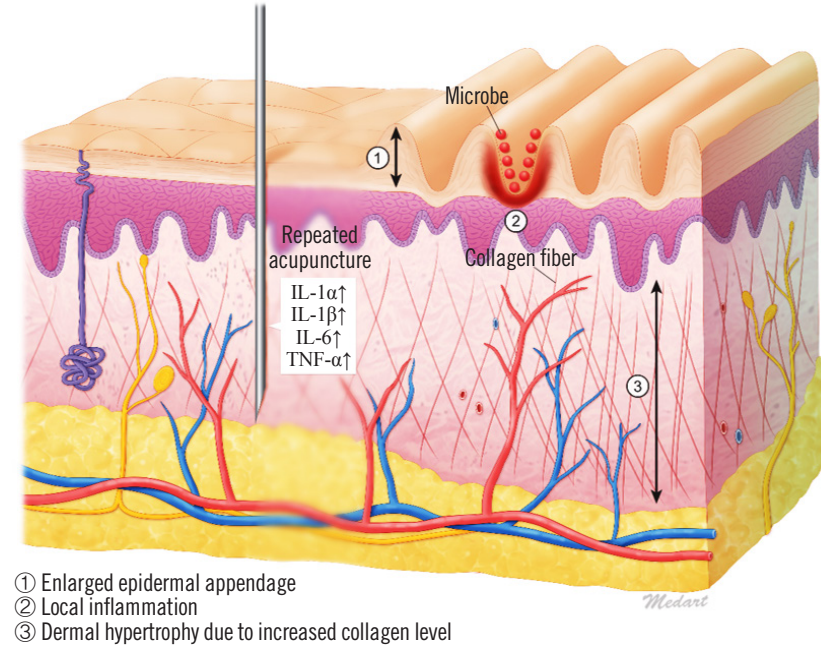

Fig. 4. Schema of our case's cutis verticis gyrata pathophysiology. IL, interleukin; TNF, tumor necrosis factor.

histological findings [3], it is important for clinicians to be aware of the characteristics of these findings.

Little is known regarding the etiology of CVG, although it is thought that the specific pathophysiological mechanism depends on the underlying condition [6]. Histologically, CVG resembles a hypertrophic scar or keloid, especially in cases of secondary CVG. In general, the reticular layer of hypertrophic scars contains inflammatory cells, an increased number of fibroblasts, newly formed blood vessels, and collagen deposits. Furthermore, proinflammatory genes in the skin are responsive to trauma, as shown by the upregulation of proinflammatory factors such as interleukin (IL)-1a, IL-1b, IL-6, and tumor necrosis factor alpha in scar tissues. The upregulation of these factors may cause chronic inflammation, which promotes invasive scar tissue growth [7]. Additionally, when skin furrows develop, complications may occur because of bacterial and mycotic superinfections in the furrows, which exacerbate chronic inflammation. We suspect that this scarring mechanism may have contributed to the case of CVG described herein (Fig. 4).

Recently, some researchers have proposed that CVG may be a genetic condition caused by an autosomal dominant mutation in the fibroblast growth factor receptor 2 (FGFR2) gene, since dermal hypertrophy is a characteristic of CVG. FGFR2 encodes a transmembrane tyrosine kinase capable of functioning as an inflammatory, angiogenic, or mitogenic factor, in accordance with the microenvironment and/or the cell type. The sequence of FGFR2 is highly homologous to that of the IL-6 promoter. Therefore, IL-6 levels are likely to be elevated in 
patients with CVG [8]. On the other hand, other authors reported the effectiveness of IGF in CVG's condition. They stated that IGF levels were elevated in cases of CVG, and that increased IGF levels were linked to the prominent presence of fibroblasts and the interstitial deposition of glycosaminoglycans [9]. In our case, however, the laboratory results were normal, which led us to suspect that chronic inflammation only due to acupuncture was the cause of CVG. The self-performed acupuncture induced repeated dermal injuries and infections, which were followed by hypersensitivity reactions and the development of hypertrophic scarring $[7,10]$. Of course, the lack of immunochemical test in tissue might remain a limitation for this study.

Several modalities have been explored to treat CVG (both primary and secondary), ranging from pharmaceutical approaches such as thyroid extract, steroids, topical antiseptics, and antihistamines to more diverse approaches such as sleep therapy, irradiation, and even psychotherapy; however, none of these approaches have been successful. The decision to perform surgical repair of CVG should be made on an individualized basis, considering the location and size of the lesion, the nature of the underlying condition, and the patient's wishes. Although CVG is a disfiguring process, it is almost always benign, with the sole exception of secondary CVG associated with cerebriform intradermal nevus, which may transform into malignant melanoma. Therefore, complete excision is the treatment of choice in such cases [1]. We also performed total excision and advancement flap coverage. The patient had favorable results after the operation and has experienced no long-term complications. However, there was a fly in the ointment; the aesthetically important eyebrow was removed after surgery. It will have to be complemented later with tattoos, etc.

In conclusion, in this report, we analyzed the overall pathophysiology of a case of secondary forehead CVG, an extremely rare condition, with a description of how we achieved good results via surgical treatment.

\section{Conflict of interest}

No potential conflicts of interest relevant to this article are reported.

\section{ORCID iDs}

Joo Sung Jung https://orcid.org/0000-0002-2515-9275

Nam Kyu Lim ～https://orcid.org/0000-0002-0964-983X

\section{References}

1. Snyder MC, Johnson PJ, Hollins RR. Congenital primary cutis verticis gyrata. Plast Reconstr Surg 2002;110:818-21.

2. Harish V, Clarke F. Isolated cutis verticis gyrata of the glabella and nasal bridge: a case report and review of the literature. J Plast Reconstr Aesthet Surg 2013;66:1421-3.

3. van Geest AJ, Berretty PJ, Klinkhamer PJ, et al. Cerebriform intradermal naevus (a rare form of secondary cutis verticis gyrata). J Eur Acad Dermatol Venereol 2002;16: 529-31.

4. Kara IG. Forehead lifting for cutis verticis gyrata. Plast Reconstr Surg 2003;111:1777-8.

5. Ulrich J, Franke I, Gollnick H. Cutis verticis gyrata secondary to acne scleroticans capitis. J Eur Acad Dermatol Venereol 2004;18:499-502.

6. Kosumi H, Izumi K, Natsuga K, et al. Cutis verticis gyrata fluctuation with atopic dermatitis disease activity. Acta Derm Venereol 2017;97:1245-6.

7. Ogawa R. Keloid and hypertrophic scars are the result of chronic inflammation in the reticular dermis. Int J Mol Sci 2017;18:606.

8. Yoo KH, Lee JW, Jang WS, et al. Cutis verticis gyrata and alopecia areata: a synchronous coincidence? Yonsei Med J 2010;51:612-4.

9. Schunter JA, Metze D, Bohm M. Is IGF-1 a key player in the pathogenesis of acromegaly-associated cutis verticis gyrata? Exp Dermatol 2017;26:85-6.

10. Park SM, Kim WJ, Mun JH, et al. Adverse events associated with acupuncture: a clinicopathologic review. Int J Dermatol 2016;55:757-63. 